\title{
New Transit Measurements of WASP 43b and HD 189733b
}

Richard P. Olenick ${ }^{1 *}$, Arthur Sweeney ${ }^{1}$, Philip Lenzen ${ }^{1}$, Samantha Garza ${ }^{1}$, Cecilia Hassan ${ }^{1}$, Gianna Milton ${ }^{1}$, Clement Say ${ }^{1}$, Stephen Flowers ${ }^{2}$, Nick Bacsewski ${ }^{2}$, Chris Littler ${ }^{2}$

\section{Abstract}

Known exoplanets not only provide excellent targets for students who are learning to acquire data with remote observatories and to process the data but also fulfill a scientific need for repeated measurements to determine the stability of known parameters. We present recent measurements taken by undergraduate students with a remotely accessed telescope at the Dark Skies Observatory Collaborative in West Texas on two well-studied exoplanets. WASP $43 \mathrm{~b}$ has a published orbital period of 0.81347753 days and its host $\mathrm{K} 7 \mathrm{~V}$ star has a visual magnitude of 12.4. HD 189733b has a published orbital period of 2.21857312 days around its K1V star of visual magnitude 7.67. Both planets orbit within the corona of their host stars and, as such, appear to experience changes in their orbital periods, transit timings, and other parameters. We examined the historical trends, combined them with our measurements in the mid-transit timings for the stars, and determined there are significant changes. Astronomersfrom college students to professionals-need this continued monitoring in order to keep system models up to date.

\section{Keywords}

planets and satellites-detection-dynamical evolution and stability-exoplanets

${ }^{1}$ University of Dallas 1845 E. Northgate Dr. Irving, TX 75062 USA

${ }^{2}$ University of North Texas 1155 Union Circle Denton, TX 76203 USA

*Corresponding author: olenick@udallas.edu

\section{Introduction}

When an exoplanet passes between its star and the Earth, it blocks out a fraction of that star's observable light in a transit of the star. From the initial ingress of the exoplanet's shadow onto the star, through its full eclipse time, to its egress from the star, the time duration of the total eclipse depends not only on the planet's orbital period, but also the radii of both the planet and star, as well as the planet's impact parameter (the distance above or below the equator of the star) and distance from the star.

The transit duration corresponds to the time an exoplanet's shadow covers any part of the star. In Fig.1, between the first and final contacts, A and B, the sector of arc $\alpha$ is the fraction of the circular orbit for which the planet eclipses the star, $\alpha / 2 \pi$. The transit duration time, $T_{\text {duration }}$ is the corresponding fraction $P \alpha / 2 \pi$ of the orbital period. Expressing $\alpha$ in terms of the stellar and planetary radii, the radius of the orbit $a$ and the impact parameter $b$, it is a simple matter of geometry and trigonometry to obtain the result shown in Eq.1 (Seager and MallenOrnelas, 2003).

$T_{\text {duration }}=P \frac{\alpha}{2 \pi}=\frac{P}{\pi} \sin ^{-1}\left(\frac{\sqrt{\left.\left(R_{*}+R_{P}\right)^{2}-\left(b R_{*}\right)^{2}\right)}}{a}\right)$ 


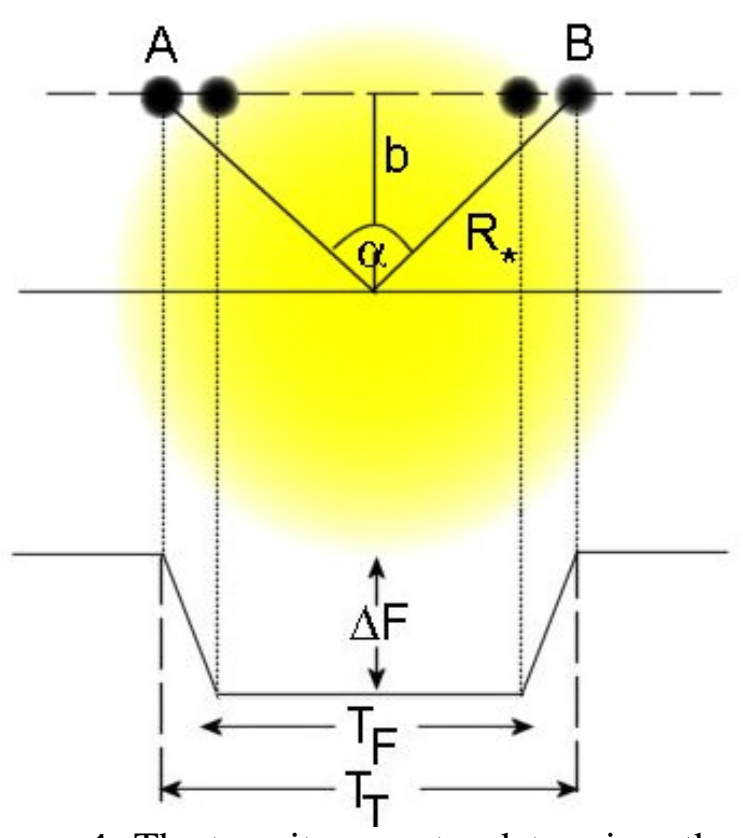

Figure 1. The transit geometry determines the transit duration which is dependent on the radius of the star $R_{*}$, the radius of the planet $R_{P}$, the impact parameter $b$, and the orbital radius $a$.

Through the measurements of these parameters, characteristics of the planet and star can be determined.

The transit is observed as a decrease in stellar flux in the light curve. A typical light curve for an exoplanet transit shows a measurable decrease in the flux on the order of about 10 - 50 mmags (millimags), or approximately $1-5 \%$ of the star's observed flux. The transit depth corresponds to the ratio of the cross-sectional areas of the planet to the star, i.e., to the ratio of their squared radii. The shape of the light curve reveals the relatively constant rise and fall of the flux in relation to the ingress and egress of the planet. Between these regions, however, the light curve generally follows a much flatter trend when the entire planet is eclipsing the star and the eclipsed area is unchanging. The midpoint of this flat region defines the midpoint time, or epoch, of the transit and is the point at which the centers of the planet and the star are observationally aligned and from which future transits are periodically predicted. The duration of the transit is manifested as the width of the well-shaped curve, from just before its ingress to after its egress. Figure 2 shows a light curve extracted from data illustrating these characteristics.

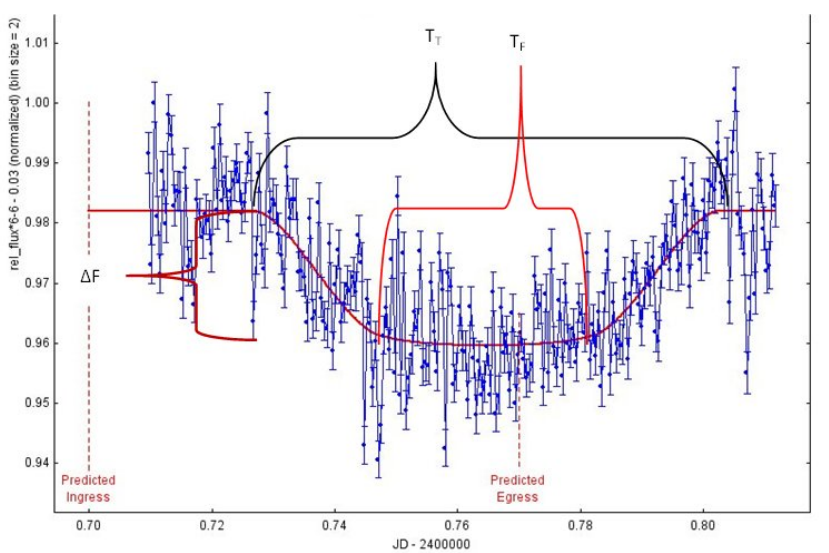

Figure 2. The shape of the light curve characteristically includes the depth and width of the transit, and its ingress, egress and epoch, thereby delineating both the planet's full eclipse and its total eclipse. In addition, the curve exhibits the effects of limb darkening.

In Fig. 2 the blue data points have a model curve fitted as a solid red line. The shape of the transit fit is not flat but curved as a result of limb darkening: At its limb the star emits less light than from the interior and so the planet blocks less light. By determining a model least-squares fit to the light curve, four key physical parameters can be measured: The planet's orbit period $P$, the transit flat time $T_{F}$ (of the full eclipse), the total transit time $T_{T}$ (of the total eclipse), and the change in stellar photon flux $\Delta F$; the last three of these are denoted in Fig. 2. The period is not immediately apparent in the light curve, but can be obtained from a periodogram analysis of the light curves from several separate transits. The total and flat transit times as well as the transit depth are measured from the model curve.

From these four parameters Seager showed that parameters can be deduced, including the semimajor axis $a$ and angle of inclination $i$ of the planet's elliptical orbit, as well as the radii $R_{P}$ and $R_{*}$ the planet and star, respectively (Seager and MallenOrnelas, 2003). Figure 3 illustrates the relationships among the measured parameters and the calculated parameters. 


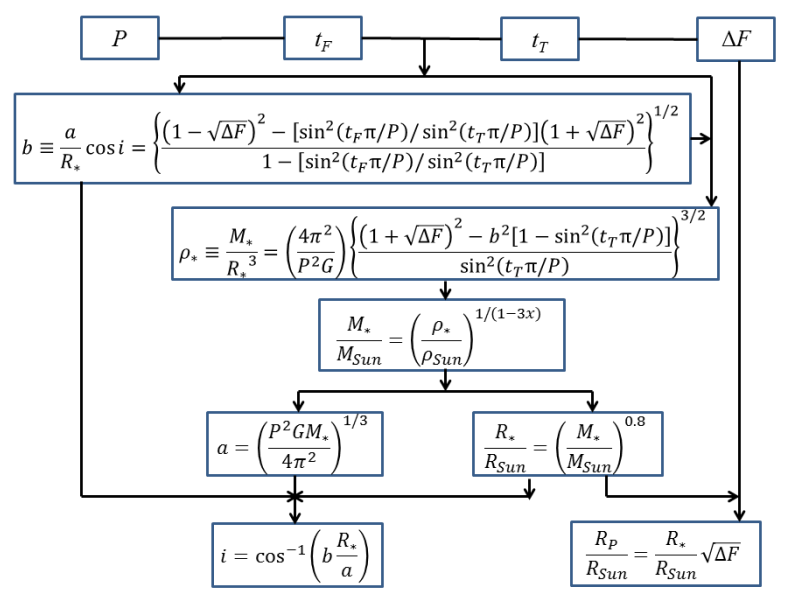

Figure 3. In measuring the period, transit flat time, total transit time, and change in flux, other key parameters can be deduced, including the planet's impact parameter, the star's mass and density, the semi-major axis and angle of inclination of the planet's orbit, and the sizes of both the planet and star. The latter four are the most important to this analysis.

\section{WASP 43b}

WASP 43b was discovered in 2011 in the Wide Angle Search for Planets (WASP) Project (Hellier et al., 2011). The planet is described as a hot Jupiter transiting a cool K7V star with a surface temperature of $4400 \mathrm{~K}$, mass of $0.6 \mathrm{M}_{\odot}$, and a 15.6 day rotational period. The hot Jupiter has a mass $m=2.052 M_{\text {Jup }}$ masses, orbital period $P=$ $0.81347753 \pm 7 \times 10^{-7}$ days, and semi-major axis $a=0.01526$ AU. WASP $43 \mathrm{~b}$ is the hot Jupiter-type exoplanet closest to its parent star and, as such, the planet is expected to be phase locked with its rotation period equaling its orbital period so that the same side always faces the star.

\section{HD 189733b}

The transiting exoplanet HD 189733b was discovered in 2005 in the constellation Vulpecula near the Dumbbell Nebula, M27 (Bouchy et al., 2005). It orbits a spectral type KV star, HD 189733, which has an apparent magnitude of $7.67 \mathrm{mag}$. Although HD 189733 is part of a binary system, its far-out companion star has an orbital period of 3,200 years and does not affect the transits of the exoplanet. At a dis- tance of 63.4 light-years from Earth, HD 189733b is the closest observable hot Jupiter to the Earth. Its mass is $m=1.142 \mathrm{M}_{J u p}$ and its semi-major axis is $a=0.03142 \mathrm{AU}$.

The most unusual feature of HD $189733 \mathrm{~b}$ is that it orbits within the star's corona, which extends out to $0.033 \mathrm{AU}$ from the star, with a period $P=$ $2.21857312 \pm 6.6 \times 10^{-7}$ days. The planet races around its star with an average velocity of about $152.5 \mathrm{~km} / \mathrm{s}$ (341,133 mph). The supersonic motion through the coronal plasma and its close proximity to the star superheat the planet to temperatures of $930{ }^{\circ} \mathrm{C}$ and creates shock waves as far out as 12.75 $R_{P}$ in front of it (Llama et al., 2013).

\section{Methods}

Observations of the transits were made at the Dark Skies Observatory Collaborative (DSOC) in West Texas, near McDonald Observatory, by remotely operating the telescope from Irving, Texas. To take the images we used an f/8 Ritchey-Chrétien 16-in telescope attached to a Bisque ME II mount, as shown in Fig.4, to which was attached an SBIG ST10 CCD camera of un-binned resolution 2184 by 1472 pixels, with a Johnson R-band filter. Because the CCD quantum efficiency peaks at 660 nanometers we used the R-band filter exclusively for our observations to maximize the camera's efficiency.

Observations were made by undergraduate students controlling the remote telescope by using TeamViewer, TheSkyX (TSX) and MaximDL (MDL) software. Approximately two hours before the predetermined start time for the transit, the DSOC control computer, and also the Bisque mount, are turned on via a switch securely accessed online. The control computer is then accessed from the Irving lab computer with the TeamViewer software, which is installed on both computers. The observatory roof is also opened to allow the telescope and cameras time to equilibrate thermally. To reduce thermal noise, the camera is set to cool continuously at $-20^{\circ} \mathrm{C}$.

Once the star has been targeted, the exposure time is determined for a signal-to-noise ratio that avoids saturating the image (saturation occurs at 


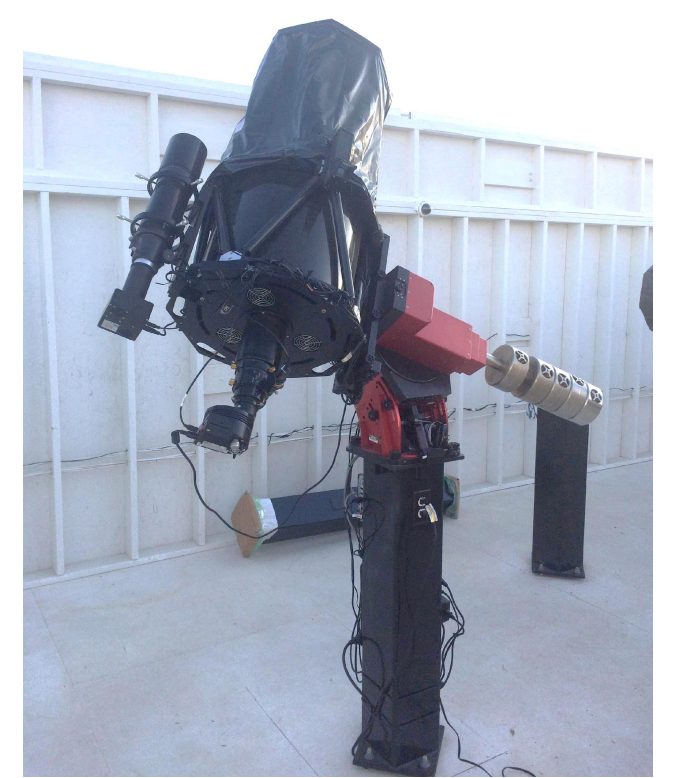

Figure 4. A Bisque ME II mount supports both the Ritchey-Chrétien 16-in telescope and the SBIG ST-10 CCD camera.

Table 1. Ephemerides for transits of WASP $43 \mathrm{~b}$ used to determine nights for observations.

$\begin{array}{llll}\text { UT date } & \text { Ingress } & \text { Mid-transit } & \text { Egress } \\ 3 / 20 / 2018 & 8.40 & 8.98 & 9.56 \\ 3 / 21 / 2018 & 3.92 & 4.5 & 5.08 \\ 3 / 25 / 2018 & 5.54 & 6.12 & 6.7 \\ 3 / 29 / 2018 & 6.16 & 7.74 & 8.32\end{array}$

about 55,000 counts). The remote setup then automatically takes data on the star throughout the transit. Flats, darks and bias images are taken for calibration.

The ephemerides were generated from data on on the Czech Exoplanet Transit Database site (Poddanỳ et al., 2010). Table 1 shows the calculated ephemerides of transits of WASP 43b occurring in March 2018. Out of those listed in the table, only the $3 / 21 / 2018$ transit was visible. At the other times the transit occurred at either too low altitude or near to the Moon. The ephemerides for the transits of HD 189733b are shown in Table 2. The 7/12/2017 and the 9/1/2017 transit of HD 189733b were successfully observed, whereas the other predicted transits were obscured either by sunlight, clouds, or rain.
Table 2. Ephemerides for transits of HD 189733b used to determine nights for observations.

$\begin{array}{llll}\text { UT date } & \text { Ingress } & \text { Mid-transit } & \text { Egress } \\ 7 / 12 / 2017 & 4.73 & 5.60 & 6.47 \\ 7 / 23 / 2017 & 6.96 & 7.83 & 8.70 \\ 8 / 1 / 2017 & 3.94 & 4.81 & 5.68 \\ 8 / 12 / 2017 & 6.17 & 7.04 & 7.91 \\ 8 / 21 / 2017 & 3.16 & 4.03 & 4.90 \\ 9 / 1 / 2017 & 5.40 & 6.27 & 7.14\end{array}$

\section{AstrolmageJ: Photometric Reduction}

Post-observation data analysis includes subtracting the calibration images (flat frames, bias frames and dark frames) from the raw data images, and, from those corrected images, creating the light curve, which is flux or magnitude as a function of recorded time. The calibration image processing, or photometric reduction, takes place with the use of the AstroImageJ (AIJ) software (Collins et al., 2017). The data processor (DP) module creates a master frame for each of the calibration types (flat, bias, and dark), which it uses to calibrate the images one by one. After the images have been calibrated, the target star as well as several comparison stars are identified. AstroImageJ automatically records the flux and a magnitude of each star using standard aperture photometry.

Once a light curve has been produced from the data, a model, or synthetic, curve is determined by a least-squares fit to the data. From the model fit, physical parameters of the transit that were described earlier can be measured. The model curve takes into account four user-specified parameters and fits seven transit parameters and multiple detrending parameters to calculate the physical planetary parameters. The orbital period and eccentricity, the argument of its periapsis, and stellar radius are the specified input values. From the model light curve the transit parameters are the raw baseline flux, the squared ratio of planet-star radii $\left(R_{P} / R_{*}\right)^{2}$, the ratio of the planet's semi-major axis of its orbit to the star's radius $a / R_{*}$, the transit midpoint or center $T_{c}$, the orbital inclination $i$, and the quadratic limb darkening curves at ingress and egress. The model curve attempts several different variations of 
these parameters, incrementing them each iteration with a step size peculiar to the parameter, until it reaches the best set of fits. The goodness of the fit is measured by the $\chi^{2} / d o f$ value, where dof is the degrees of freedom. For an ideal fit $\chi^{2} /$ dof should be approximately one. Further work with different combinations of the detrending parameters, as well as fitting the transit parameters for different set values, yield improved values for $\chi^{2} / d o f$.

\section{Use of Historical Data and O-C Analysis}

Another important method in this work centers on the use of historical data to extract changes in the periods, transit depths, and transit widths of known exoplanets. We use the Czech Exoplanet Transit Database (ETD) from which data on well-known transits that have been contributed by numerous observers can be downloaded and analyzed (Poddanỳ et al., 2010). The quality of the data in the database is ranked from 1 to 5 , where 1 represents data with the smallest uncertainties. With multiple values of transit midpoints, it is possible to perform an $\mathrm{O}-\mathrm{C}$ analysis, which calculates the difference between the observed mid-transit times and the calculated mid-transit times obtained from knowledge of the orbital period. In order to calculate O-C for a known period $P$, the product of the cycle number $n$ and the period, that is, the calculated value, is subtracted from the measured (observed) mid-transit time. Equation 2 gives the $\mathrm{O}-\mathrm{C}$ calculation where $E$ is the starting observed mid-transit time, $n$ is the cycle number, and $P$ is the orbital period of the exoplanet.

$$
O-C=H J D_{\text {mid }}-(n P+E)
$$

\section{Results}

\section{WASP 43b Period}

The light curve obtained for WASP $43 \mathrm{~b}$ for the $2 / 21 / 2018$ transit is shown in Fig. 5. The $2 \times 2$ binned data are plotted in blue along with their uncertainties and the transit model curve is shown in black. Shown in red is the light curve of a comparison star. The predicted ingress and egress of the transit are also indicated as per the ephemerides. The best fit parameters from the model curve of Fig. 5 are given in Table 3.

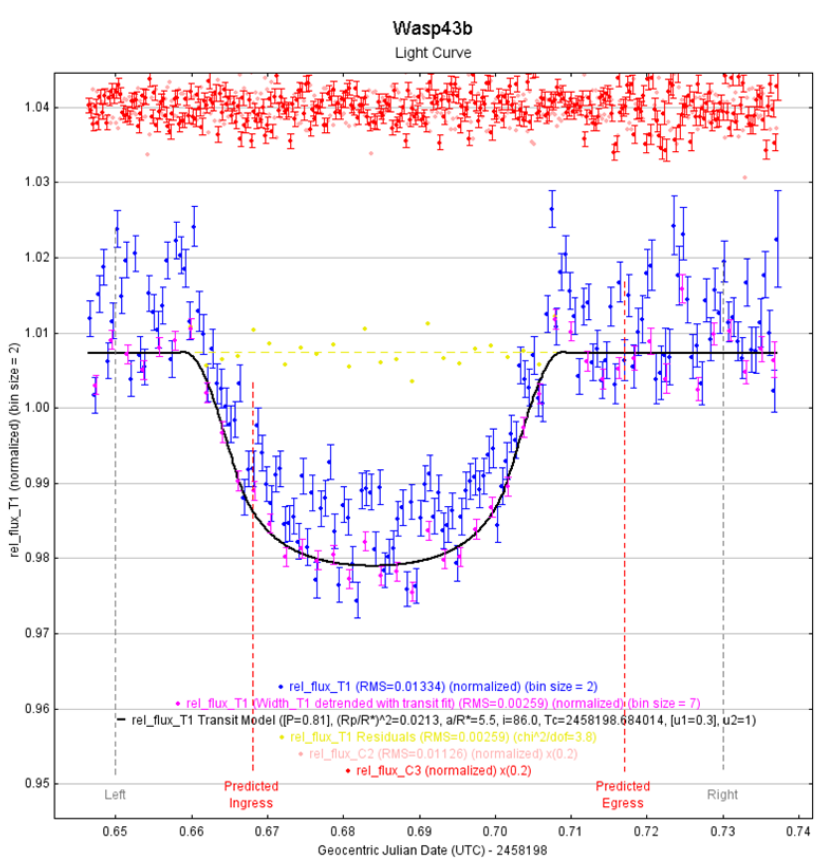

Figure 5. Processed light curve for WASP $43 \mathrm{~b}$ showing the $2 \times 2$ binned data points in blue and the best fitted model curve is shown in black.

Table 3. Transit Fit for $03 / 21 / 2018$ of WASP $43 b$

\begin{tabular}{ll}
\hline Parameter & Best Fit \\
\hline Raw baseline flux & 0.574172383 \\
$\left(R_{P} / R_{*}\right)^{2}$ & 0.021258953 \\
$a / R_{*}$ & 5.548254771 \\
$T_{C}$ & 2458198.684013694 \\
Inclination $i$ & 85.95 \\
Quad LD $u 1$ & 0.3000 \\
Quad LD $u 2$ & 0.9999 \\
\hline
\end{tabular}

We combined six years of historical data from the Czech Exoplanet Transit Database (Poddany et al., 2010) with our data to construct a graph of $\mathrm{O}-\mathrm{C}$ for the mid-transit times as a function of observation date. The resulting graph is shown in Fig. 6 . The graph reveals a negative slope, which indicates a possible anomaly-that the initially accepted period for the exoplanet is not consistent with observations. The best straight line fit is found to be 


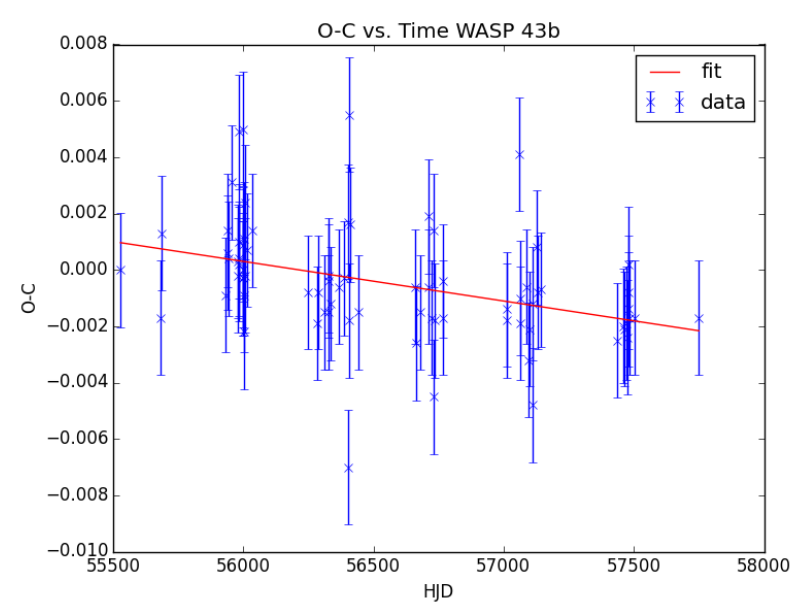

Figure 6. The observed minus calculated (O-C) transit midpoints for WASP $43 \mathrm{~b}$ are graphed in blue as a function of time from historical data and our measurements. A linear fit to the data is shown in red. The negative slope of the fitted line indicates a period for the starting epoch that is too small.

$$
O-C=0.07909288-0.00000141 \times H J D_{\text {mid }}
$$

with a $\chi^{2} / d o f$ of 0.868 . The fitted curve yields a correction of $1.15 \times 10^{-6}$ day or $0.0017 \mathrm{~min}$ to the period of WASP $43 \mathrm{~b}$. This anomaly found in the period is therefore on the order of 1 millionth of the period and is within our experimental uncertainty of the orbital period. Hoyer et al. also used a compilation of data to determine that the change in the orbit of WASP $43 \mathrm{~b}$ is $P_{d o t}=\frac{d P}{d t}=1.5 \pm 7.3 \mathrm{~ms} / \mathrm{yr}$, which is also consistent with a constant orbital period, and rules out orbital decay of the exoplanet (Hoyer et al., 2016).

\section{HD 189733b Transit Period O-C vs. Epoch}

The processed light curve for HD 189733b is shown in Fig. 7 and the values extracted from the fit are given in Table 4. Light curve analysis and modeling produce planetary parameters, which, when combined with auxiliary historical data, allow for inferences to be drawn regarding the orbital evolution of HD 189733b. The inclusion of historical data is necessary in order to observe the accumulation of small changes over a very long time period; the Exoplanet

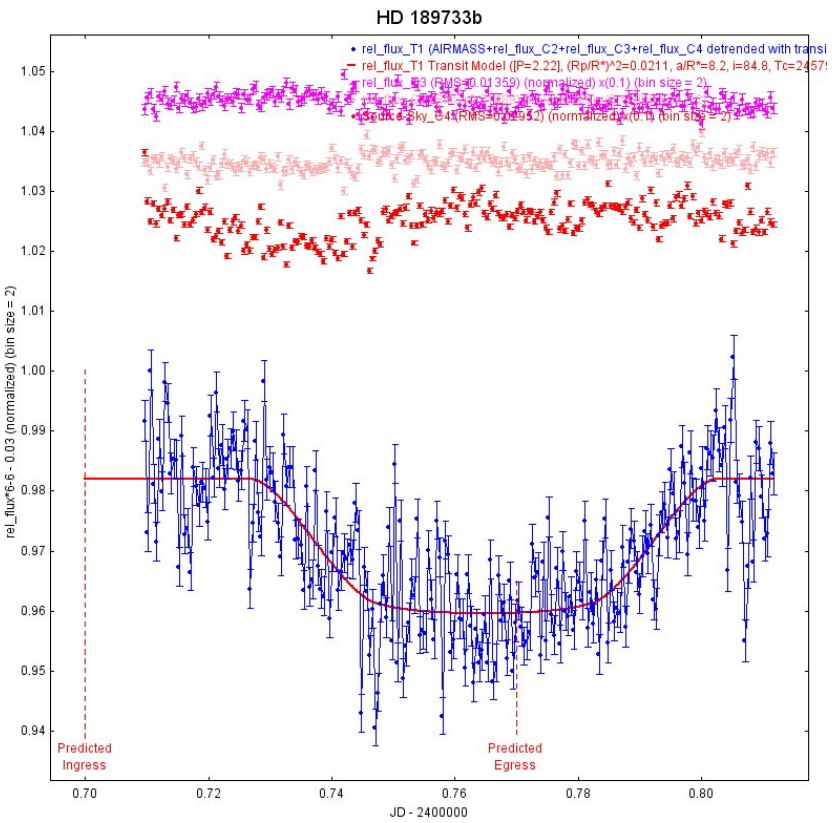

Figure 7. Processed light curve for HD 189733b showing the $2 \times 2$ binned data points in blue and the best fitted curve in red.

Transit Database has recorded data for HD 189733b for as far back as September 2005, and continues, currently, up to September 2016 (Poddanỳ et al., 2010). By including our measurements with all these measurements, the transit evolution of HD 189733 b can be extended back a full twelve years, and changes can be deduced that are predictive of the planet's future evolution.

Table 4. Transit Fit for 07/12/2017 of HD 189733 b

\begin{tabular}{ll}
\hline Parameter & Best Fit \\
\hline Raw baseline flux & 8.477195017 \\
$\left(R_{P} / R_{*}\right)^{2}$ & 0.024323961 \\
$a / R_{*}$ & 8.644306433 \\
$T_{C}$ & 2457997.762563860 \\
Inclination $i$ & 85.51 \\
Quad LD $u 1$ & 0.947585262 \\
Quad LD $u 2$ & -0.243269972 \\
AIRMASS & 0.007914396 \\
\hline
\end{tabular}

Measurable changes are found in the mid-transit times, as shown in Fig. 8 in which $\mathrm{O}-\mathrm{C}$ values are plotted as a function of cycle number. Leastsquares fits to the data were made. We use the 
Akaike Information Criterion (AIC) to determine if a parabolic fit is a better or worse-fitting model of the data than a linear fit. The AIC is calculated from

$$
A I C=N \log (R S S / N)+2 k
$$

where $N$ is the number of observations, $k$ the number of model parameters, and RSS the residual of the sum of squares for the fit. We find that the quadratic fit gives a lower AIC and indicates a better fit of the data. A parabolic fit of the form $A x^{2}+B x+C$, yields the coefficients shown in Table 5 . We made fits for both the best of the historical data, Ranks 1-2, (see the first three rows in Table 5) which included our data and also for all the data (Table 5 second three rows. The fitted curve shown in red in Fig. 8 is for all the data. We discuss the meaning of negative coefficient for the quadratic term in the next section.

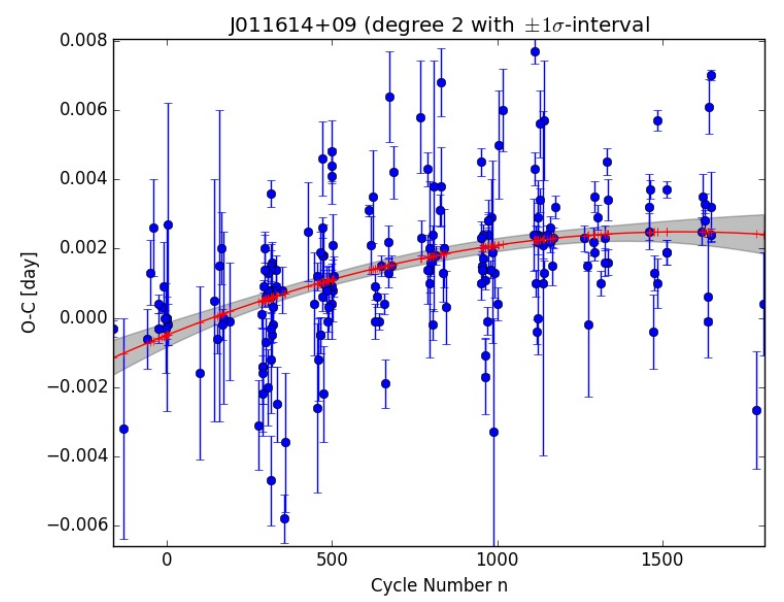

Figure 8. The graph includes both the historical data and the 7/12/17 and 9/1/17 transit data, plotting them against a $\pm 1 \sigma$ deviation band. Though period calculations for O-C normally fall on the order of hundredths, these points are only thousandths.

\section{Discussion}

Several researchers have shown that precise measurements of transit time variations of exoplanets can be sensitive to other planetary bodies, such as
Table 5. Summary of Curve Fitting Parameters

\begin{tabular}{lr}
\hline \multicolumn{2}{c}{ O-C: Parabolic Fit, Ranks 1-2 } \\
\hline A & $-1.2283 \mathrm{E}-09 \pm 5.8368 \mathrm{E}-10$ \\
B & $3.8315 \mathrm{E}-06 \pm 9.7849 \mathrm{E}-07$ \\
C & $-5.0421 \mathrm{E}-04 \pm 3.5170 \mathrm{E}-04$ \\
\hline O-C: Parabolic Fit, Ranks 1-5 \\
\hline A & $-1.6325 \mathrm{E}-09 \pm 4.4875 \mathrm{E}-10$ \\
B & $0.0000 \pm 0.0000$ \\
C & $-0.0002 \pm 0.0003$
\end{tabular}

exo-moons. In addition, transit timing variations of the exoplanets closest to their host stars can provide tests of tidal dissipation theory (Watson and Marsh, 2010). Researchers also have evidence of period changes which are attributed to mass loss by exoplanets. For example, Linsky analyzed HD 209458b using spectroscopic data (Linsky et al., 2010). Exospheric heating leading to mass loss due to X-ray and extreme ultraviolet radiation have also been estimated (Ehrenreich and Désert, 2011). Evidence of a slow period change due to frictional drag and subsequent mass loss by an exoplanet traveling with the corona of its star has also been presented (Jiang et al., 2016). Since WASP 43b is known to have such an intracoronal orbit, it would be expected that its period should be changing. The historical data plotted in Fig. 6 shows, however, a period that is consistent with it being constant over time. The least squares fit to the data suggests that any period anomaly is within $0.0017 \mathrm{~min}$ out of a period of 0.8134775 days. The observed variations in mid-transit times for WASP 43b were found to be within the uncertainty in measurement of the orbital period. The question that remains is why the period is constant even though WASP $43 \mathrm{~b}$ is definitely orbiting within its star's corona.

\section{Projection of HD 189733b Orbital Dynam- ics}

Figure 8 plots the deviation between the observed and calculated mid-transit times for many transits over several cycles. In such an O-C diagram the shape reveals information: If the graph is linear, the slight error in the epochal period is simply propa- 
gating over multiple transits, causing the increased magnitude of deviation. If the graph is parabolic, however, the deviations are accelerating, and therefore, the orbital period is evolving. This can be attributed to a nonzero rate-of-change of the planet's mass. The negative value of the quadratic coefficient $(A)$ for the graph in Fig. 8 reveals a decreasing period. The best fit to the data is parabolic (with a negative value for $A$ ) for any collection of ranks of the data, as indicated in Table 5. The uncertainty in the quadratic coefficient (A) indicates that better historical data is nonetheless needed.

Orbital mechanics predicts several significant parameter changes attributable to a negative quadratic coefficient $A$ for the $\mathrm{O}-\mathrm{C}$ curve. The first of these is the rate-of-change of the period. Since $A$ is negative, the rate-of-change is also negative and it is given by

$$
\begin{aligned}
P_{d o t} & =\frac{d P}{d t}=\frac{2 A}{P}=-\left(2.08 \times 10^{-10}\right. \\
& \left.= \pm 9.88 \times 10^{-11}\right) \frac{d a y s}{d a y}
\end{aligned}
$$

Thus the period is decreasing at a rate of $2.08 \times$ $10^{-10}$ days per day. The parameter also describes a non-conservative mass loss from the planet of mass $m$ which is given by

$$
\begin{aligned}
m_{d o t} & =\frac{P_{d o t}}{P} \frac{m+M_{*}}{2} \approx \frac{P_{d o t}}{P} \frac{m_{*}}{2} \\
& =-\left(1.44 \times 10^{-5} \pm 6.95 \times 10^{-6}\right) \frac{M_{J u p}}{y r}
\end{aligned}
$$

Thus the planet is losing mass at a rate of $1.44 \times$ 10-5 Jupiter masses per year. For the case of a circular orbit, which is approximately the case for HD 189733 b given that its eccentricity is 0.0041 , and for constant total mass of the star-planet system, the rate-of-change of the semi-major axis, $a_{d o t}$, decreases proportionally to the period's rate-of-change:

$$
\begin{aligned}
a_{d o t} & =\frac{d a}{d t}=\frac{2}{3} \frac{P_{d o t}}{P} a \\
& =-\left(0.20 \times 10^{-13} \pm 6.24 \times 10^{-14}\right) \frac{A U}{y r} .
\end{aligned}
$$

Therefore we can conclude that the planet is spiraling inwards at a rate of $9.20 \times 10^{-13}$ AU per year. This conclusion implies that the planet will crash into the star when its current semi-major axis reaches the radius of the star. That time can be calculated by integrating Eq. 7:

$$
\int_{a_{0}}^{R_{*}} \frac{d a}{a}=c \int_{0}^{t} d t
$$

where the constant is $c=\frac{2}{3} \frac{P_{d o t}}{P}$. This results in

$$
t=\frac{1}{c} \ln \left(\frac{R_{*}}{a_{0}}\right)
$$

where $a_{0}$ is the initial orbital radius of the exoplanet.

Using the values determined from our analysis, the time for HD $189733 \mathrm{~b}$ to crash into its star is $t_{\text {fallintostar }}=(93 \pm 45)$ million years. Improved and additional data could further verify this prediction, but these orbital dynamics are the best for the current data. Numerical simulations conclude that tidal disruption of the orbit of HD $189733 \mathrm{~b}$ would lead to an unstable orbit on a timescale in $1.0 \times 10^{9} \mathrm{yr}$ (Levrard et al., 2009). Other dissipative mechanisms, such as the intracoronal motion of the planet must be invoked to explain the decay of the planetary orbit on the order of millions of years.

\section{Conclusion}

Both WASP 43b and HD 189733 b orbit within the coronas of their host stars and, as such, would be expected to experience changes in their orbital periods, transit timings, and other parameters. We examined the historical trends and combined them with our measurements of the mid-transit times for transits about the two stars. We found no significant period change for WASP $43 \mathrm{~b}$ but a measurable change in the period of HD 189733b. Although both planets have intracoronal orbits, why one exoplanet exhibits a decaying orbit but the other does not is a subject for further research. Investigation of closein exoplanets - those with intracoronal orbits - may reveal more facets of their orbital evolution and time scales.

We find that undergraduate students can learn how to operate a telescope remotely, acquire quality data, conduct the reduction of the raw data into 
light curves, and fit models to the data that allow significant scientific conclusions to be drawn.

Although data presented in this work only contributes to previous knowledge, it inspires curiosity to discover more about exoplanets. Taking and analyzing data on known exoplanets is also a way in which amateur astronomers and students can "check up" on parameters of exoplanets to see if any parameters have changed and to keep models up to date.

\section{Acknowledgments}

Support from the Donald A. Cowan Institute at the University of Dallas provided telescope time at the Dark Skies Observatory Collaborative. The authors also acknowledge the sustained support of the Joe Neuhoff Fund for Astronomy at the University of Dallas that made this work possible.

\section{References}

Bouchy, F., Udry, S., Mayor, M., Moutou, C., Pont, F., Iribarne, N., Da Silva, R., Ilovaisky, S., Queloz, D., Santos, N., et al. (2005). ELODIE metallicity-biased search for transiting hot jupiters-II. a very hot jupiter transiting the bright k star HD 189733. Astronomy \& Astrophysics, 444(1):L15-L19.

Collins, K. A., Kielkopf, J. F., Stassun, K. G., and Hessman, F. V. (2017). AstroImageJ: image processing and photometric extraction for ultraprecise astronomical light curves. The Astronomical Journal, 153(2):77.

Ehrenreich, D. and Désert, J.-M. (2011). Massloss rates for transiting exoplanets. Astronomy \& Astrophysics, 529:A136.

Hellier, C., Anderson, D., Cameron, A. C., Gillon, M., Jehin, E., Lendl, M., Maxted, P., Pepe, F., Pollacco, D., Queloz, D., et al. (2011). WASP43b: The closest-orbiting hot Jupiter. Astronomy \& Astrophysics, 535:L7.

Hoyer, S., Pallé, E., Dragomir, D., and Murgas, F. (2016). Ruling out the Orbital Decay of the
WASP-43b Exoplanet. The Astronomical Journal, 151(6):137.

Jiang, G., Lai, C.-Y., Savushkin, A., Mkrtichian, D., Antonyuk, K., Griv, E., Hsieh, H.-F., and Yeh, L.-C. (2016). The Possible Orbital Decay and Transit Timing Variations of the Planet WASP43b. The Astronomical Journal, 151(1):17.

Levrard, B., Winisdoerffer, C., and Chabrier, G. (2009). Falling transiting extrasolar giant planets. The Astrophysical Journal Letters, 692(1):L9.

Linsky, J. L., Yang, H., France, K., Froning, C. S., Green, J. C., Stocke, J. T., and Osterman, S. N. (2010). Observations of mass loss from the transiting exoplanet HD 209458b. The Astrophysical Journal, 717(2):1291.

Llama, J., Vidotto, A., Jardine, M., Wood, K., Fares, R., and Gombosi, T. (2013). Exoplanet transit variability: bow shocks and winds around HD 189733b. Monthly Notices of the Royal Astronomical Society, 436(3):2179-2187.

Poddanỳ, S., Brát, L., and Pejcha, O. (2010). Exoplanet Transit Database. Reduction and processing of the photometric data of exoplanet transits. New Astronomy, 15(3):297-301.

Seager, S. and Mallen-Ornelas, G. (2003). A unique solution of planet and star parameters from an extrasolar planet transit light curve. The Astrophysical Journal, 585(2):1038.

Watson, C. and Marsh, T. (2010). Orbital period variations of hot Jupiters caused by the Applegate effect. Monthly Notices of the Royal Astronomical Society, 405(3):2037-2043. 